\title{
Geleia de buriti (Mauritia flexuosa): agregação de valor aos frutos do cerrado brasileiro
}

\author{
Buriti (Mauritia flexuosa) jelly: adding value to fruits from the Brazilian savanna
}

\author{
Lismaíra Gonçalves Caixeta Garcia', William Fagundes Guimarães², Ester Candido Rodovalho², \\ Nayara Ribeiro Alves de Avelar Peres², Fernanda Salamoni Becker², Clarissa Damiani* \\ 1 Universidade Federal de Goiás (UFG), Departamento de Agronomia, Goiânia/GO - Brasil \\ 2 Universidade Federal de Goiás (UFG), Departamento de Engenharia de Alimentos, Goiânia/GO - Brasil
}

\section{${ }^{*}$ Corresponding Author:}

Clarissa Damiani, Universidade Federal de Goiás (UFG), Departamento Engenharia de Alimentos, Av. Esperança, s/n, Campus Samambaia, CEP: 74690-900, Goiânia/GO - Brasil, e-mail: damianiclarissa@hotmail.com

Cite as: Buriti (Mauritia flexuosa) jelly: adding value to fruits from the Brazilian savanna. Braz. J. Food Technol., v. 20, e2016043, 2017.

\section{Resumo}

O buriti é uma palmeira brasileira que se destaca pela sua beleza e pelos seus múltiplos usos na alimentação humana, apresentando elevado valor nutricional. Visando ao seu maior aproveitamento, foi realizada a elaboração de geleia de buriti. O produto foi caracterizado em seu aspecto físico-químico, por meio de análises de: composição proximal, fibras, valor energético, sólidos solúveis totais, $\mathrm{pH}$, acidez total titulável, potencial antioxidante e vitaminas lipossolúveis. A geleia apresentou potencial antioxidante, além de consideráveis teores de vitaminas $A$ e $D$, podendo também ser considerada fonte de fibras. Logo, o processamento de buriti, na forma de geleia, é mais uma alternativa para o seu aproveitamento e para a agregação de valor ao fruto do cerrado, além de este possuir valor nutricional.

Palavras-chave: Potencial antioxidante; Vitamina A; Processamento.

\section{Abstract}

Buriti is a Brazilian palm that stands out for its beauty and its multiple uses as a food for humans, with high nutritional value. Aiming to maximize its exploitation, buriti jelly was produced. A physicochemical characterization of the product was carried out by determining the proximate composition, fibre, energy, total soluble solids, $\mathrm{pH}$, titratable acidity, soluble vitamins and the antioxidant potential. The jelly showed potential antioxidant activity, as well as considerable amounts of vitamins $\mathrm{A}$ and $\mathrm{D}$, and can also be considered a source of fibre. Thus, the processing of buriti in the form of jelly is one more alternative for the exploitation and value aggregation of a Brazilian savanna fruit, as well as having nutritional value.

Keywords: Antioxidant potential; Vitamin A; Processing.

\section{Introdução}

O buriti (Mauritia flexuosa) é considerado como a palmeira mais abundante e naturalmente presente no bioma amazônico do Brasil. Desempenha um papel importante na conservação da fauna, uma vez que seus frutos servem como fonte de alimento para muitas espécies de pássaros e mamíferos. Além disso, é utilizado para a produção de bebidas e alimentos de forma caseira (VIEIRA et al., 2010). A polpa de buriti possui quantidades consideráveis de carotenoides, polifenóis e ácido ascórbico, apresentando potencial para ser usado na prevenção de doenças causadas pelo estresse oxidativo (AGUIAR; SOUZA, 2017).

Estudos têm demonstrado efeitos benéficos do consumo de frutas, os quais têm sido atribuídos à presença de nutrientes, como as vitaminas A, C e E (DE OLIVEIRA E SILVA et al., 2012; GAO et al., 2012; WANG et al., 2012), e principalmente ao conteúdo de compostos bioativos encontrados nos vegetais (FLORES et al., 2012; LI et al., 2013). Muitos desses compostos 
atuam como sequestradores de radicais livres, enquanto outros agem como quelantes de metais catalisadores de reações de geração de espécies reativas de oxigênio (HALLIWELL; GUTTERIDGE, 2007; FLOEGEL et al., 2011).

O desenvolvimento de novos produtos com elevadas proporções de frutas em suas formulações e com boas propriedades funcionais e nutricionais contribui para diversificar as possibilidades de mercado, principalmente, se os produtos forem atrativos, práticos e com maior vida-de-prateleira (MARTíN-ESPARZA et al., 2011a,b). Devido à diversidade das frutas existentes no território brasileiro e ao fato de que estas apresentam propriedades adequadas para o processamento, além de propriedades funcionais, demonstra-se que este é um mercado que tem potencial de crescimento no Brasil.

A produção de geleia, a partir da polpa de buriti, é uma alternativa de aproveitamento dos frutos, possibilitando sua utilização de forma comercial, maior oferta no mercado e qualidade de comercialização. O processamento de geleia é interessante, pois exige poucos equipamentos e traz, como vantagens para o setor produtivo, o aproveitamento de frutas impróprias para a comercialização in natura, em compota ou desidratada. Também permite o uso do excedente da produção (LOPES, 2007). Diante do exposto, objetivou-se, com o presente trabalho, desenvolver e caracterizar geleia de buriti, a fim de aproveitar o fruto, bem como avaliar suas propriedades nutricionais.

\section{Material e métodos}

\subsection{Obtenção dos frutos e elaboração da geleia}

Os frutos de buriti foram colhidos maduros, no Cerrado Goiano e, em seguida, foram selecionados (retirando impurezas), lavados em água corrente e sanitizados com solução de hipoclorito de sódio a $100 \mu \mathrm{L} \mathrm{L}^{-1}$, por 15 minutos.

Para elaboração da geleia, foi utilizada a polpa de buriti, com o pH inicial corrigido para 3,2 com ácido cítrico, para obter geleificação adequada e realçar o sabor natural da fruta; pectina comercial, para compensar a deficiência no conteúdo natural da fruta, e sacarose. Inicialmente, adicionou-se à polpa um terço do açúcar, homogeneizou-se a mistura e mediu-se o teor de sólidos solúveis, sendo adicionada água potável até a redução para $20{ }^{\circ}$ Brix. Posteriormente, a solução foi submetida ao aquecimento, até início da ebulição, momento no qual se adicionou mais um terço do açúcar, previamente homogeneizado com a pectina. Após nova ebulição, adicionou-se o restante do açúcar e esperou-se concentrar até $60^{\circ}$ Brix. Em seguida, foi adicionado ácido cítrico, diluído em água, e concentrou-se a mistura até o teor de sólidos solúveis de $65^{\circ}$ Brix. Para o envase da geleia, utilizaram-se potes de vidro sextavados de $230 \mathrm{~mL}$, previamente sanitizados com solução de hipoclorito de sódio a $200 \mu \mathrm{L} \mathrm{L}^{-1}$, por
15 minutos. O produto foi submetido à exaustão, resfriado e armazenado à temperatura ambiente $\left( \pm 28^{\circ} \mathrm{C}\right)$.

\subsection{Caracterização físico-química}

Para a caracterização físico-química, foram realizadas análises de composição proximal (umidade, cinzas, lipídeos, proteínas e carboidratos totais), fibra alimentar total, solúvel e insolúvel, sólidos solúveis totais, acidez total titulável e pH (AOAC, 2010). O valor energético foi calculado por meio da utilização dos coeficientes de ATWATER (carboidratos $=4,0 \mathrm{kcal} \mathrm{g}^{-1} ;$ lipídeos $=9,0 \mathrm{kcal} \mathrm{g}^{-1}$; proteínas $\left.=4,0 \mathrm{kcal} \mathrm{g}^{-1}\right)$ (MERRILL; WATT, 1973).

O potencial antioxidante foi determinado nos extratos etéreo, etanólico e aquoso, pelo método do DPPH, utilizando-se (2,2 difenil-1-picrilhidrazil), segundo Brand-Williams et al. (1995), com as modificações propostas por Borguini e Torres (2009). As vitaminas lipossolúveis $(A, D, E$ e K) foram determinadas por cromatografia líquida de alta eficiência (HPLC), sendo separadas na mesma coluna em uma única corrida aplicando-se eluição isocrática com metanol como fase móvel. Todas as análises foram realizadas em triplicas e os resultados foram expressos por meio de médias e desvio padrão.

\section{Resultados e discussão}

A composição proximal da geleia de buriti está apresentada na Tabela 1. A legislação brasileira (BRASIL, 1978) estabelece teor máximo de umidade de

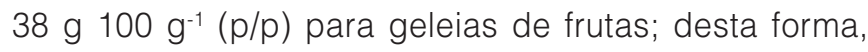
a geleia de buriti apresentou-se dentro dos padrões aceitáveis da legislação.

Celestino (2013), ao analisar a composição proximal de geleia à base de buriti, encontrou valores

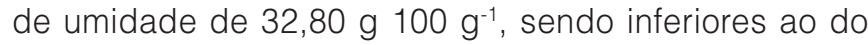
presente trabalho. Quanto aos teores de carboidratos, fibra alimentar (Tabela 2) e proteínas, Celestino (2013)

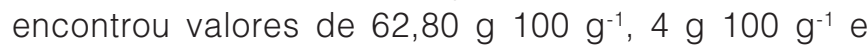
$0,20 \mathrm{~g}^{100 \mathrm{~g}^{-1}}$, respectivamente, sendo estes inferiores ao do presente trabalho. Em relação ao teor de cinzas,

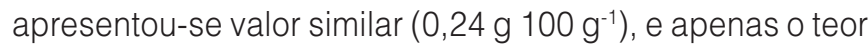

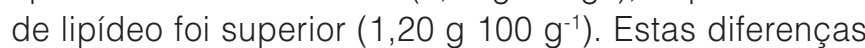
na composição proximal podem ser resultado tanto dos ingredientes utilizados e da forma de processamento quanto da variedade, do local de cultivo, da temperatura, do tipo de solo, entre outros fatores do local de cultivo do buriti, fatores estes que podem alterar a composição do fruto.

Analisando-se o teor de fibras da geleia de buriti (Tabela 2), nota-se que esta pode receber alegação de produto funcional, pois, segundo a Agência Nacional de Vigilância Sanitária (BRASIL, 1999), a alegação de alimento funcional pode ser utilizada desde que a porção do produto pronto para o consumo forneça, no mínimo, $3 \mathrm{~g}$ de fibras, se o alimento por sólido, e 1,5 g de fibras, se o alimento 
por líquido. A fibra alimentar torna-se importante, pois não contribui com nenhuma caloria para a nossa dieta, mas os metabólitos liberados pelas bactérias no cólon, depois de ingeridas as fibras, são usados por seres humanos e outros mamíferos para satisfazer as suas necessidades energéticas (TURNER; LUPTON, 2011).

A geleia de buriti apresentou maior teor de fibra insolúvel, quando comparada com a fibra solúvel. A fibra insolúvel aumenta o volume das fezes pela sua própria massa e, também, pela água que a mantém ligada ou adsorvida, sendo benéfica no tratamento da constipação, da síndrome do intestino irritável e da doença diverticular (TARPILA et al., 2005). Por outro lado, sabe-se que as fibras solúveis são caracterizadas pela capacidade de aumentar a viscosidade e de reduzir a resposta glicêmica e níveis plasmáticos de colesterol (ELLEUCH et al., 2011). O valor energético da geleia de buriti deve-se, quase que exclusivamente, ao açúcar presente no buriti e ao açúcar utilizado no processamento, uma vez que o produto apresentou valores relativamente baixos de proteínas e lipídeos.

O teor de sólidos solúveis da geleia de buriti apresentou-se dentro dos padrões aceitáveis da legislação brasileira, que estabelece valores entre $62^{\circ}$ Brix e $65^{\circ} \mathrm{Brix}$,

Tabela 1. Valores médios e desvio padrão dos teores de umidade, cinzas, proteínas, lipídeos e carboidratos.

\begin{tabular}{cc} 
Macronutriente & $\begin{array}{c}\text { Valores médios }\left(\mathbf{g} \mathbf{1 0 0} \mathbf{~ g}^{-1}\right) \\
\pm \text { desvio padrão }\end{array}$ \\
\hline Umidade & $35,31 \pm 0,4994$ \\
Cinzas & $0,25 \pm 0,0000$ \\
Proteínas & $0,38 \pm 0,0057$ \\
Lipídeos & $0,94 \pm 0,0351$ \\
Carboidratos & $63,12 \pm 0,47$ \\
\hline
\end{tabular}

para geleia de frutos. De acordo com Lopes (2007), o pH ótimo para formação de gel é de 3,0 a 3,2, sendo que, em valores acima de 3,4, pode não ocorrer geleificação. No presente trabalho, foram encontrados valores superiores de $\mathrm{pH}$, porém este valor não alterou a estrutura do gel. Carneiro et al. (2015), ao elaborarem geleia de physalis, observaram que, mesmo com altos valores de $\mathrm{pH}(4,35)$, o produto apresentou textura e cor características de geleia, possibilitando aceitação do produto.

Os valores de acidez total titulável encontrados na geleia de buriti (Tabela 2) estão de acordo com Torrezan (1998), que afirma que a acidez total da geleia deve estar entre 0,5 e 0,8\%, pois, acima de 1\%, pode ocorrer sinérese. Hansen (2011) elaborou geleia de mangaba e observou redução da acidez total titulável durante 90 dias de armazenamento, de 0,76\% para 0,72\%. Conforme Oliveira et al. (2013), a acidez fornece uma informação valiosa do estado de conservação do alimento, pois, quando este entra em processo de decomposição, a concentração de íons de hidrogênio apresenta-se quase sempre alterada.

O processo de extração, utilizando solventes com diferentes polaridades, possibilitou a extração de compostos antioxidantes em quantidades variadas (Tabela 2). Observou-se que o extrato etanólico exibiu maior potencial antioxidante, quando comparado aos extratos etéreo e aquoso. Estas diferenças mostram que, para a extração seletiva de antioxidantes naturais, é importante e necessário um estudo sobre o solvente mais apropriado. Os diversos solventes certificam a máxima solubilização dos antioxidantes presentes na amostra. A utilização de três solventes de diferentes polaridades - éter etílico $(2,9)$, etanol $(5,2)$ e água destilada (9) - possibilita a solubilização de compostos mais polares (extrato aquoso), de polaridade intermediária (extrato etanólico) e apolares (extrato etéreo) (BORGUINI; TORRES, 2009). No entanto, de uma forma

Tabela 2. Características físico-químicas e valor energético de geleia de buriti.

\begin{tabular}{|c|c|}
\hline Análise & Média \pm desvio padrão \\
\hline Fibra solúvel $\left({\left.\mathrm{g} 00 \mathrm{~g}^{-1}\right)}^{-1}\right.$ & $1,00 \pm 0,0002$ \\
\hline Fibra insolúvel (g $\left.100 \mathrm{~g}^{-1}\right)$ & $3,53 \pm 0,0001$ \\
\hline Fibra total $\left(\mathrm{g}_{\left.100 \mathrm{~g}^{-1}\right)}\right.$ & $4,53 \pm 0,0002$ \\
\hline Valor calórico (kcal) & $262,46 \pm 2,1200$ \\
\hline Sólidos solúveis totais ( $\left.{ }^{\circ} \mathrm{Brix}\right)$ & $62,00 \pm 0,0000$ \\
\hline $\mathrm{pH}$ & $3,64 \pm 0,0000$ \\
\hline Acidez total titulável $\left(\mathrm{g} 100 \mathrm{~g}^{-1}\right)$ & $0,60 \pm 0,0000$ \\
\hline Potencial antioxidante - extrato etéreo (\% descoloração) & $5,00 \pm 0,0003$ \\
\hline Potencial antioxidante - extrato etanólico (\% descoloração) & $9,13 \pm 0,0001$ \\
\hline Potencial antioxidante - extrato aquoso (\% descoloração) & $8,69 \pm 0,0001$ \\
\hline Vitamina A (mg $\left.100 \mathrm{~g}^{-1}\right)$ & $1,41 \pm 0,0002$ \\
\hline Vitamina D $\left(m g 100 \mathrm{~g}^{-1}\right)$ & $1,20 \pm 0,0002$ \\
\hline Vitamina $E\left(m g 100 \mathrm{~g}^{-1}\right)$ & $0,80 \pm 0,0001$ \\
\hline Vitamina $\mathrm{K}\left(\mathrm{mg} 100 \mathrm{~g}^{-1}\right)$ & $0,51 \pm 0,0000$ \\
\hline
\end{tabular}


geral, observou-se que a geleia de buriti apresentou baixa atividade antioxidante devido à baixa porcentagem de descoloração do DPPH. Esta baixa atividade pode ser justificada pela degradação dos compostos bioativos durante o processamento da geleia.

Dentre as vitaminas analisadas na geleia de buriti, a vitamina A é a que se encontrou em maior concentração (Tabela 2). A vitamina A é um importante nutriente, destacando-se por sua função na integridade cutânea, na proteção de estruturas e funções oculares, no sistema imunológico e na redução da morbimortalidade por doenças infecciosas, como diarreia, sarampo e malária. Sua carência pode levar a problemas oculares, como xeroftalmia e cegueira noturna (QUEIROZ et al., 2013). Ainda segundo Rodriguez-Amaya (2010), a deficiência deste micronutriente é a segunda maior do mundo, sendo, portanto, importante estimular seu consumo.

A segunda maior concentração de vitaminas foi creditada à vitamina $D$, seguida pelas vitaminas $E$ e $\mathrm{K}$. A vitamina D, também chamada de colecalciferol, é um hormônio esteroide, cuja principal função consiste na regulação da homeostase do cálcio e na formação e reabsorção óssea, através da sua interação com as paratireoides, os rins e os intestinos (ARNSON et al., 2007), sendo, assim, de grande importância para o ser humano.

\section{Conclusão}

A geleia de buriti apresenta potencial antioxidante, vitaminas $A$ e D, além de consideráveis teores de fibras. Desta forma, a utilização da polpa de buriti na fabricação de geleias é uma alternativa viável tecnológica e nutricionalmente.

\section{Referências}

AGUIAR, J. P. L.; SOUZA, F. C. A. Desidratação e pulverização de polpa de buriti (Mauritia flexuosa L.): avaliação da vida-deprateleira. Revista Brasileira de Fruticultura, v. 39, p. 1-7, 2017. Número especial.

ARNSON, Y.; AMITAL, H.; SHOENFELD, Y. Vitamin D and autoimmunity: new etiological and therapeutic considerations.

Annals of the Rheumatic Diseases, v. 66, n. 9, p. 1137-1142, 2007. PMid:17557889. http://dx.doi.org/10.1136/ard.2007.069831.

ASSOCIATION OFFICIAL ANALYTICAL CHEMISTS - AOAC. Official methods of analysis of the Association of Official Analytical Chemists. 18th ed. Gaithersburg: AOAC, 2010.

BORGUINI, R. G.; TORRES, E. A. F. S. Tomatoes and tomato products as dietary sources of antioxidants. Food Reviews International, v. 25, n. 4, p. 313-325, 2009. http://dx.doi. org/10.1080/87559120903155859.

BRAND-WILLIAMS, W.; CUVELIER, M. E.; BERSER, C. Use of a free radical method to evaluate antioxidant activity. Lebensmittel-
Wissenschaft + Technologie, v. 28, n. 1, p. 25-30, 1995. http:// dx.doi.org/10.1016/S0023-6438(95)80008-5.

BRASIL. Agencia Nacional de Vigilância Sanitária. Resolução CNNPA n 12, 24 de julho de 1978. Aprova normas técnicas especiais relativas a alimentos (e bebidas), para efeito em todo território brasileiro. Diário Oficial [da] República Federativa do Brasil, Brasília, DF, 24 jul. 1978.

BRASIL. Agência Nacional de Vigilância Sanitária. Resolução nº 18, de 30 de abril de 1999. Aprova o Regulamento Técnico que estabelece as diretrizes básicas para análise e comprovação de propriedades funcionais e ou de saúde alegadas em rotulagem de alimentos. Diário Oficial [da] República Federativa do Brasil, Brasília, DF, 30 abr. 1999.

CARNEIRO, F.; CONTERNO, P.; LONGHI, S. A. T.; LOSS, E. M. S.; SOTILES, A. R.; RIBAS, M. F. Geleia de physalis: análise físico-química e sensorial. In: CONGRESSO DE CIÊNCIA E TECNOLOGIA DA UNIVERSIDADE TECNOLÓGICA FEDERAL DO PARANÁ - CAMPUS DOIS VIZINHOS, 3., 2015, Dois Vizinhos, Anais... Dois Vizinhos: UTFPR-DV, 2015. p. 388-389.

CELESTINO, S. M. C. Desenvolvimento e avaliação da vida de prateleira de uma geleia de buriti. Planaltina: Embrapa Cerrados, 2013. 27 p. (Boletim de Pesquisa e Desenvolvimento, 313).

DE OLIVEIRA E SILVA, A. M.; VIDAL-NOVOA, A.; BATISTAGONZÁlEZ, A. E.; PINTO, J. R.; PORTARI MANCINI, D. A.; REINA-URQUIJO, W.; MANCINI-FILHO, J. In vivo and in vitro antioxidant activity and hepatoprotective properties of polyphenols from Halimeda opuntia (Linnaeus) Lamouroux. Redox Report, v. 17, n. 2, p. 47-53, 2012. PMid:22564347. http://dx.doi.org/10 1179/1351000212Y.0000000003.

ELLEUCH, M.; BEDIGIAN, D.; ROISEUX, O.; BESBES, S.; BLECKER, C.; ATTIA, H. Dietary fibre and fibre-rich by-products of food processing: characterisation, technological functionality and commercial applications: a review. Food Chemistry, v. 124, n. 2, p. 411-421, 2011. http://dx.doi.org/10.1016/j.foodchem.2010.06.077.

FLOEGEL, A.; KIM, D. O.; CHUNG, S. J.; KOO, S. I.; CHUN, O. K. Comparison of ABTS/DPPH assays to measure antioxidant capacity in popular antioxidant-rich US foods. Journal of Food Composition and Analysis, v. 24, n. 7, p. 1043-1048, 2011. http://dx.doi.org/10.1016/j.jfca.2011.01.008.

FLORES, G.; DASTMALCHI, K.; DABO, A. J.; WHALEN, K.; PEDRAZA-PENALOSA, P.; FORONJY, R. F.; D'ARMIENTO, J. M.; KENNELLY, E. J. Antioxidants of therapeutic relevance in COPD from the neotropical blueberry Anthopterus wardii. Food Chemistry, v. 131, n. 1, p. 119-125, 2012. PMid:22363097. http:// dx.doi.org/10.1016/j.foodchem.2011.08.044

GAO, Y.; LI, C.; YIN, J.; SHEN, J.; WANG, H.; WU, Y.; JIN, H. Fucoidan, a sulfated polysaccharide from brown algae, improves cognitive impairment induced by infusion of Abeta peptide in rats. Environmental Toxicology and Pharmacology, v. 33, n. 2, p. 304-311, 2012. PMid:22301160. http://dx.doi.org/10.1016/j. etap.2011.12.022. 
Geleia de buriti (Mauritia flexuosa): agregação de valor aos frutos do cerrado brasileiro

Garcia, L. G. C. et al.

HALLIWELL, B.; GUTTERIDGE, J. Free radicals in biology and medicine. New York: OUO Oxford, 2007.

HANSEN, O. A. S. Agregação de valor aos frutos da mangabeira (Hancornia speciosa Gomes): desenvolvimento e avaliação da estabilidade de néctar e geleia. 2011. 188 f. Dissertação (Mestrado em Ciências Agrárias)-Centro de Ciências Agrárias, Ambientais e Biológicas, Universidade Federal do Recôncavo da Bahia, Cruz das Almas, 2011.

LI, F.; LI, S.; LI, H.-B.; DENG, G.-F.; LING, W.-H.; WU, S.; XU, X.-R.; CHEN, F. Antiproliferative activity of peels, pulps and seeds of 61 fruits. Journal of Functional Foods, v. 5, n. 3, p. 1298-1309, 2013. http://dx.doi.org/10.1016/j.jff.2013.04.016.

LOPES, R. L. T. Dossiê técnico-fabricação de geléias. Belo Horizonte: Fundação Centro Tecnológico de Minas Gerais CETEC, 2007. 30 p.

MARTÍN-ESPARZA, M. E.; ESCRICHE, I.; PENAGOS, L.; MATÍNEZNAVARRETE, N. Quality stability assessment of a strawberry-gel product during storage. Journal of Food Process Engineering, v. 34, n. 2, p. 204-223, 2011a. http://dx.doi.org/10.1111/j.17454530.2008.00349.x.

MARTÍN-ESPARZA, M. E.; ESCRICHE, I.; PENAGOS, L.; MATÍNEZNAVARRETE, N. Significance of osmotic temperature treatment and storage time on physical and chemical properties of a strawberr-gel product. Journal of the Science of Food and Agriculture, v. 91, n. 5, p. 894-904, 2011b. PMid:21384357. http://dx.doi.org/10.1002/jsfa.4262.

MERRILL, A. L.; WATT, B. K. Energy value of foods: basis and derivation. Washington: United States Department of Agriculture, 1973. (Agriculture Handbook, 74).
OLIVEIRA, E. S.; ANDRADE, C. K. O.; PINTO, M. S. C.; GALDINO, P. O.; TARGINI, L. C.; MEDEIROS, A. C.; SILVA, R. A.; MARACAJA, P. B. Qualidade de méis de Apis mellifera produzidos no sertão paraibano. Informativo Técnico do Semi-Árido, v. 7, n. 1, p. 203-208, 2013.

QUEIROZ, D.; PAIVA, A. A.; PEDRAZA, D. F.; CUNHA, M. A. L.; ESTEVES, G. H.; LUNA, J. G.; DINIZ, A. S. Deficiência de vitamina A e fatores associados em crianças de áreas urbanas. Revista de Saúde Pública, v. 47, n. 2, p. 248-256, 2013. PMid:24037351. http://dx.doi.org/10.1590/S0034-8910.2013047002906.

RODRIGUEZ-AMAYA, D. B. Quantitative analysis, in vitro assessment of bioavailability and antioxidant activity of food carotenoids-A review. Journal of Food Composition and Analysis, v. 23, n. 7, p. 726-740, 2010. http://dx.doi.org/10.1016/j.jfca.2010.03.008.

TARPILA, A.; WENNBERG, T.; TARPILA, S. Flaxseed as a functional food. Current Topics in Nutraceutical Research, v. 3, n. 3, p. 167-188, 2005.

TORREZAN, R. Manual para a produção de geléias de frutas em escala industrial. Rio de Janeiro: EMBRAPA - CTAA, 1998. 27 p.

TURNER, N. D.; LUPTON, J. R. Dietary fiber. Advances in Nutrition, v. 2, n. 2, p. 151-152, 2011. PMid:22332044. http:// dx.doi.org/10.3945/an.110.000281.

VIEIRA, R. F.; AGOSTINI-COSTA, T. S.; SILVA, D. B.; SANO, S. M.; FERREIRA, F. R. Frutas nativas da região Centro-oeste do Brasil. Brasília: Embrapa Informações Tecnológica, 2010. 322 p.

WANG, J.; WANG, F.; YUN, H.; ZHANG, H.; ZHANG, Q. Effect and mechanism of fucoidan derivatives from Laminaria japonica in experimental adenine-induced chronic kidney disease. Journal of Ethnopharmacology, v. 139, n. 3, p. 807-813, 2012. PMid:22210052. http://dx.doi.org/10.1016/j.jep.2011.12.022. 